\title{
A Rare Case of a Giant Retroperitoneal Gastrointestinal Stromal Tumor of Malignant Potential
}

\author{
Abdullah M. Alshamrani ", Meshare A Albattah, Rasha A Bssas, Fatemah Y Alhedaithy, Mshaeel H Almutairi, \\ Mohayad A Bkheite, Salih H Albishri
}

General Surgery Department, Security Forces Hospital Program, Riyadh, Saudi Arabia

*Corresponding author: Dr. Abdullah M. Alshamrani; Alshamraniabdullah92@gmail.com

Received 28 December 2020;

Accepted 12 January 2021;

Published 15 January 2021

\begin{abstract}
Gastrointestinal stromal tumors (GIST) are mesenchymal tumors that originate in the gastrointestinal tract. We present the case of a 30-year-old man who presented with progressive abdominal distension of one-years' duration. A physical examination revealed a distended, tense, nontender abdomen, and initial imaging investigations revealed a huge cystic, septate abdominal mass. A provisional diagnosis was made of a huge retroperitoneal, malignant, cystic mass. However, aspiration cytology showed signs suggestive of a benign cyst. Cystic tumor excision performed with control of drain site to prevent leakeage throughout the procedure. The tumor extending into the pelvis, measuring approximately $17 \times 31 \times 42 \mathrm{~cm}$ in diameter and weighing $3.78 \mathrm{~kg}$. The tumor was resected, and a histopathological examination showed findings consistent with a GIST of mixed spindle and epithelioid type. Further evaluations revealed that the tumor was positive for CD 117 and CD 34 , prompting the oncology team to start the patient on oral imatinib $400 \mathrm{mg}$ once daily. No evidence of local recurrence or distant metastasis was noted on an abdominal computed tomography scan performed during the follow-up visit up to October 2020 last follow-up. The clinical presentation of GISTs is often complex, making its diagnosis challenging, especially in patients who present with non-specific gastrointestinal symptoms. The successful management of these tumors requires a multidisciplinary approach, and regular follow-ups.
\end{abstract}

Keywords: Gastrointestinal stromal tumor, mesenchymal tumor, molecular markers, therapy

\section{Introduction}

Gastrointestinal stromal tumors (GISTs) were rarely recognized before the year 2000; however, advances in detecting mutations in the gene c-kit have led to increased recognition of these tumors ${ }^{[1]}$. They represent approximately $3 \%$ of gastrointestinal tumors, with $50-70 \%$ and $20-30 \%$ identified in the stomach and small intestine, respectively ${ }^{[1]}$. These tumors have also been reported to originate from the omentum, mesentery, or retroperitoneal space.

The most common presenting symptoms of GIST include abdominal pain or distension, vomiting, anorexia, and weight loss. Sometimes, patients with GISTs may report bleeding, leading to anemia and its consequences. In some cases, patients are asymptomatic, especially those with small-sized tumors ${ }^{[2]}$. A single report describes a case of huge GIST of the stomach that protruded into the upper abdomen ${ }^{[3]}$. Herein, we report a rare case of a giant retroperitoneal GIST extending into the pelvis in a patient who experienced progressive abdominal distension.

\section{Case Presentation}

On December 2014, A 30-year-old Saudi man with no relevant medical history presented to the general surgery clinic with a complaint of progressive abdominal distension that evolved during a one-year period. The patient assumed he was gaining weight and attempted lifestyle modifications by modifying his diet. The patient has significant history of weight loss of approximately $20 \mathrm{~kg}$ over six months.

However, his abdomen gradually increased in size. He denied a change in bowel habit. He also reported no nausea, vomiting, or urinary symptoms. A systemic review of the patient's surgical and family history was unremarkable.

On examination, the patient was conscious and oriented, and his vital signs were stable. An abdominal examination showed a distended, tense, non-tender abdomen that was dull on percussion. He was admitted for initial laboratory investigations, including a complete blood count, liver function and renal function tests, and coagulation profile; the results of the tests were unremarkable. Serum levels of the following tumor markers were measured: carcinoembryonic antigen (CEA) $1.05 \mathrm{ng} / \mathrm{ml}$ (normal), alpha-fetoprotein (AFP) $1.37 \mathrm{ng} / \mathrm{ml}$ (normal), cancer antigen 125 (CA-125) 137.7 U/ml, and CA19-9 3.36 U/ml (normal).

An ultrasound examination of the upper abdomen revealed a huge, cystic, septate, irregular, solid mass with fine internal echoes. The mass displaced the liver and spleen upward, pushed the kidneys posteriorly, and displaced the bowel loops in the bilateral flanks and the bladder inferiorly and anteriorly. A color flow Doppler revealed vascularity in the wall, septa, solid 
component of the mass. The liver parenchyma was uniform, and it was of normal size with no focal lesion.

A spiral computed tomography (CT) scan of the chest, abdomen, and pelvis with oral and intravenous contrast showed a huge, abdominal, cystic, septate mass extending into the pelvis. The contrast-enhanced mass, which measured approximately $17 \mathrm{x}$ $31 \times 42 \mathrm{~cm}$, was irregular and had nodular foci. It displaced the liver, spleen, and stomach upward and the urinary bladder downward and anteriorly. Bowel loops were also displaced in the flank. The liver, bowel, and spleen appeared normal in size, and no retroperitoneal adenopathy was observed. Also, no bowel thickening or dilation was noted. However, a small amount of ascitic fluid was observed around the liver and spleen. In the chest cavity, no mediastinal or hilar lymphadenopathy was noted (Figure1). Based on these findings, a provisional diagnosis was made of a huge retroperitoneal, malignant, cystic mass. An ultrasound-guided aspiration by intervention radiology from the cystic component of the mass was performed, 13 liters of hemorrhagic fluids were drained, and an analysis of consistency of the fluid was unremarkable; however, aspiration cytology showed signs suggestive of a benign cyst, with no epithelial or mesothelial cells noted.

Laparoscopic exploration of the abdomen was performed, and it revealed a huge abdominal mass with massive adhesions. Furthermore, a small jejunal part adhered to the tumor, and there were dilated abnormal vessels with several collaterals. Consequently, a midline laparotomy was performed. With control aspiration done through the cystic component of the tumor. Laparotomy revealed tumor was adhesive to small bowel and urinary bladder. The tumor originated mainly on the left side of the pelvic cavity. The tumor was mobile Proximally, and its undersurface could be reached. The tumor was above retroperitoneal space and just went between urinary bladder and rectum and the tumor appeared to be originating from there .Both right and left ureters identified and safeguarded .A frozen section was sent for pathological evaluation, which showed a hemorrhagic cyst and necrotic foci in the cyst wall. No epithelial cells were observed. Another frozen section obtained from the other side of the tumor showed a pelvic mass with spindle cell neoplasm. At this stage, the tumor found toughly adhered to the urinary bladder. Urology team was consulted, the urinary bladder inflated with 500 cc normal saline through foly's catheter. Dissection was done slowly down in the area of lower bladder just down to the prostatic level. The tumor was shaved from the bladder wall, and was completely removed. The small bowel loops adhered to tumor was resected around $10 \mathrm{~cm}$ from upper jejunum. The tumor was completely removed and which weighed approximately $3.78 \mathrm{~kg}$. The postoperative course was uneventful.

On day 1 postoperative, the patient started taking sips of water to transition to a liquid diet. An abdominal drain yielded of serous fluid. On day 5 postoperative, the patient was discharged and followed-up two weeks later at the general surgery clinic, looking well and tolerating a regular diet. The result of the histopathology examination showed findings consistent with a Gastrointestinal stromal tumors (GIST) of mixed spindle and epithelioid type. The tumor measured approximately $42 \mathrm{~cm}$ in longest diameter and had a mitotic activity of $<2$ mitoses / 50 high power field. Occasional foci of necrosis and hemorrhage were observed. Poor prognostic findings included the large size of the tumor, presence of necrosis, hemorrhage, atypia, and cellularity.

Patient was referred to the oncology department where further evaluations revealed that the tumor was positive for CD 117 and CD 34. He was started on oral imatinib $400 \mathrm{mg}$ once daily. No evidence of local recurrence or distant metastasis was noted on a CT scan performed during a follow-up visit six months (Figure 2). A follow-up was schedule CT of the chest, abdomen, and pelvis was suggested to be performed annually. Up to date the patient follow-up with No evidence of local recurrence or distant metastasis was noted on serial CT scan performed for the last 5 years (2015-2020). (Figure 3)

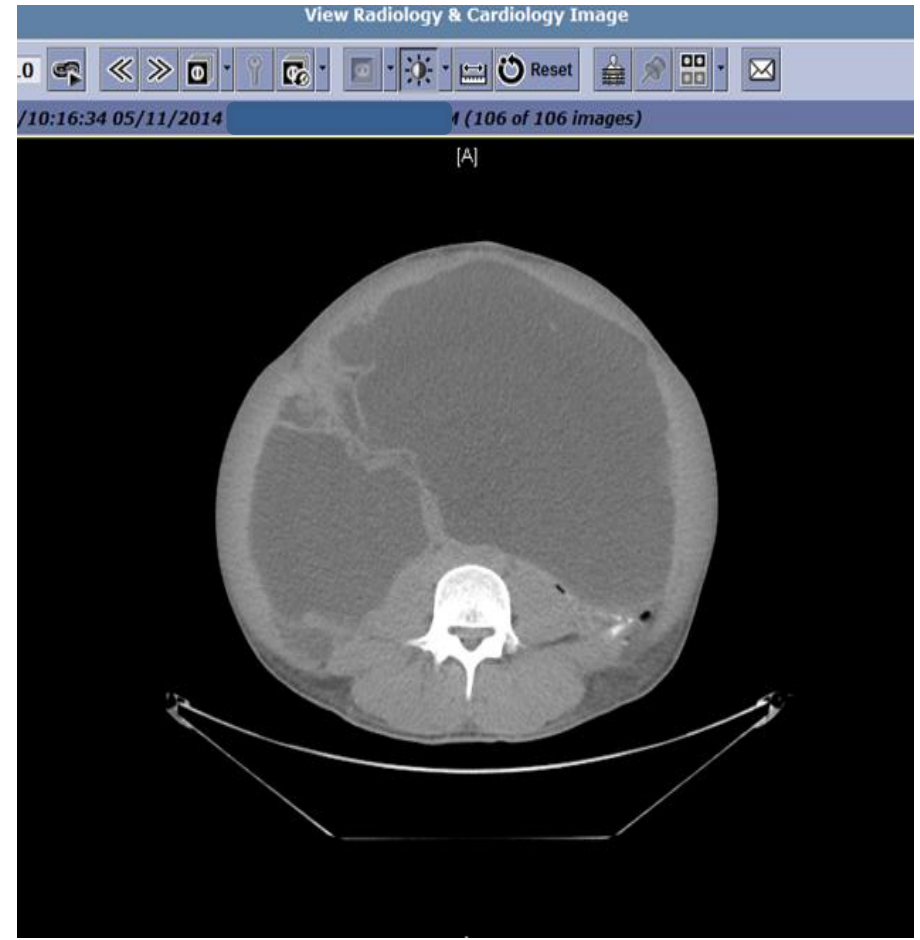

(A)

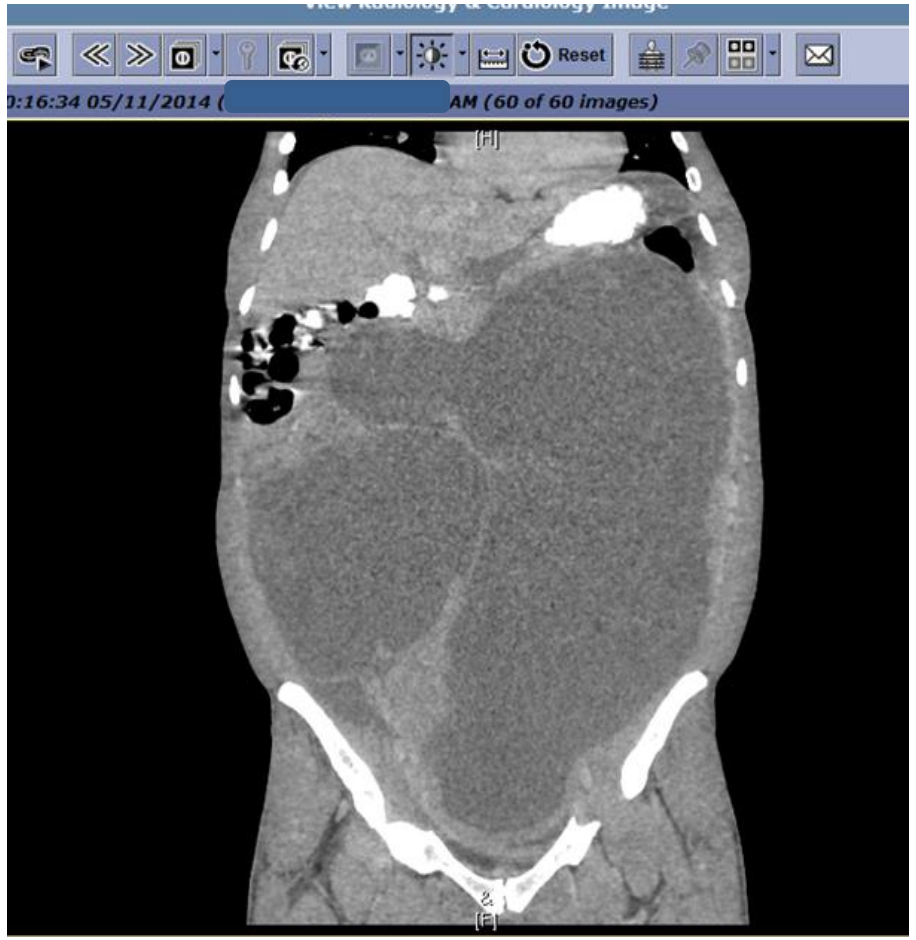

(B) 


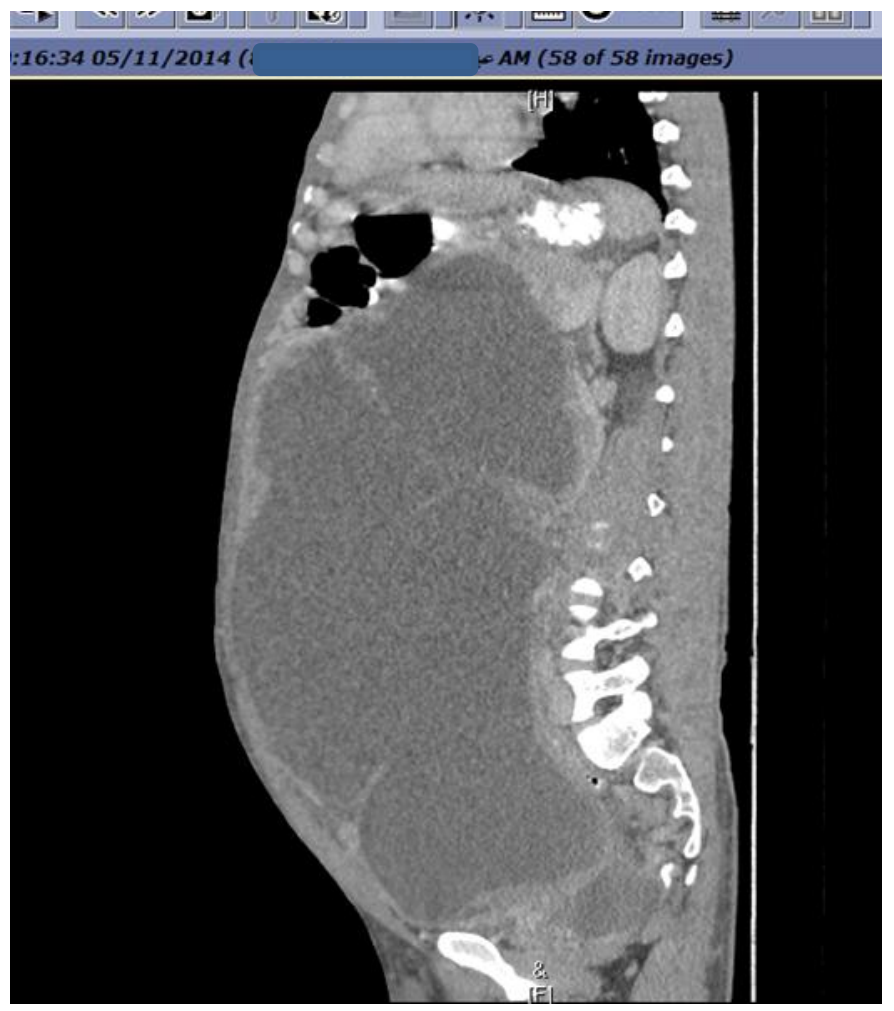

(C)

Figure 1: Axial (A), sagittal (B), and coronal (C) computed tomography sections of the chest, abdomen, and pelvis with oral and intravenous contrast showing a huge, cystic abdominal mass extending into the pelvis. The enhanced mass, measuring $17 \times 31 \times 42 \mathrm{~cm}$, is septate and has an irregular, nodular soft tissue component. It is displacing the liver, spleen, and stomach upward and the urinary bladder downward and anteriorly. The bowel loops are also displaced in the flanks.

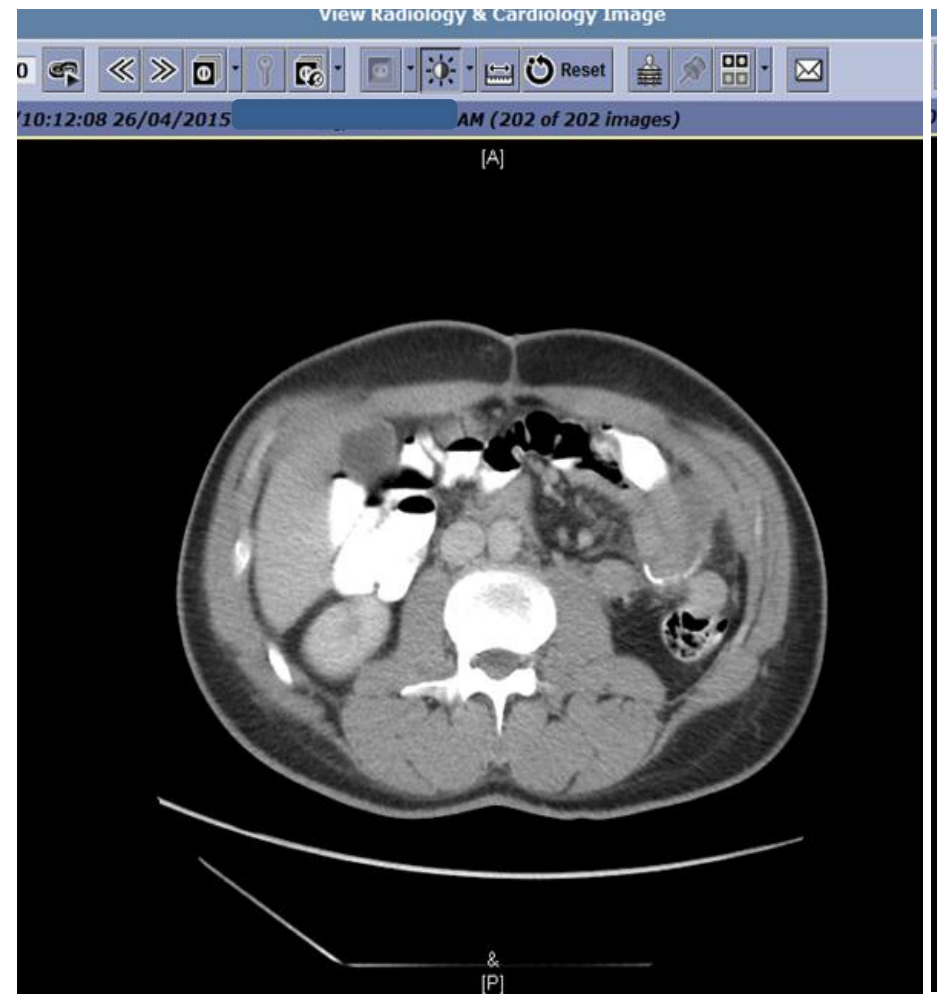

(A)

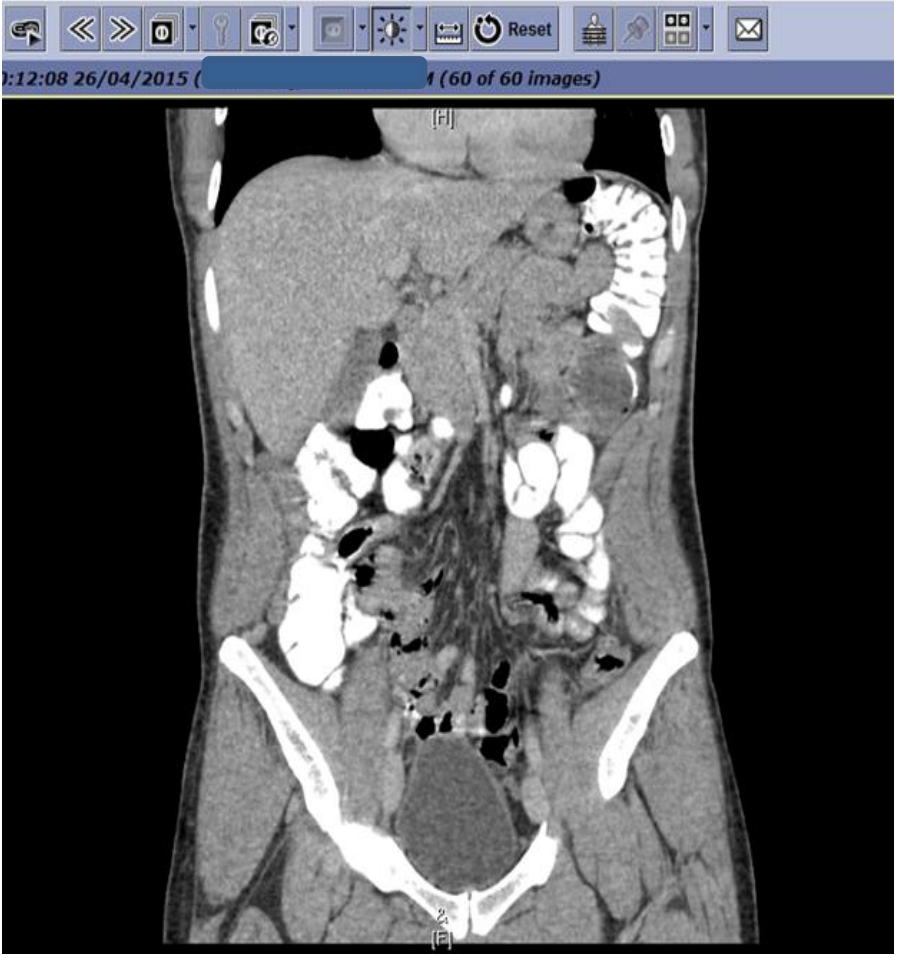

(B) 


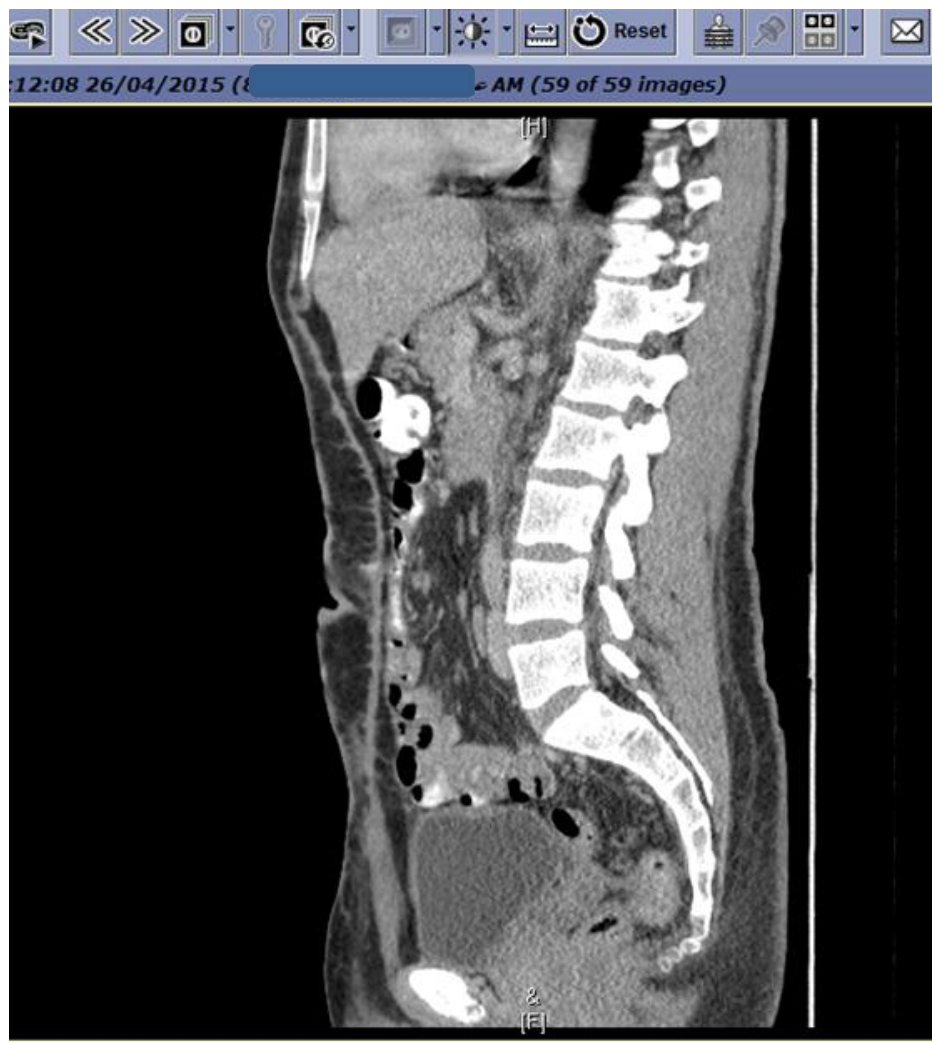

(C)

Figure 2: follow-up computed tomography scan of the abdomen. The surgical excision site appears unremarkable. There is no evidence of nodularity, thickening, or abnormal enhancement to suggest local recurrence. The retroperitoneum appears grossly unremarkable, with no signs of lymphadenopathy. The pelvic organs are intact, and there is no sign suggestive of an aggressive bony lesion. Additionally, there is no evidence of local recurrence.

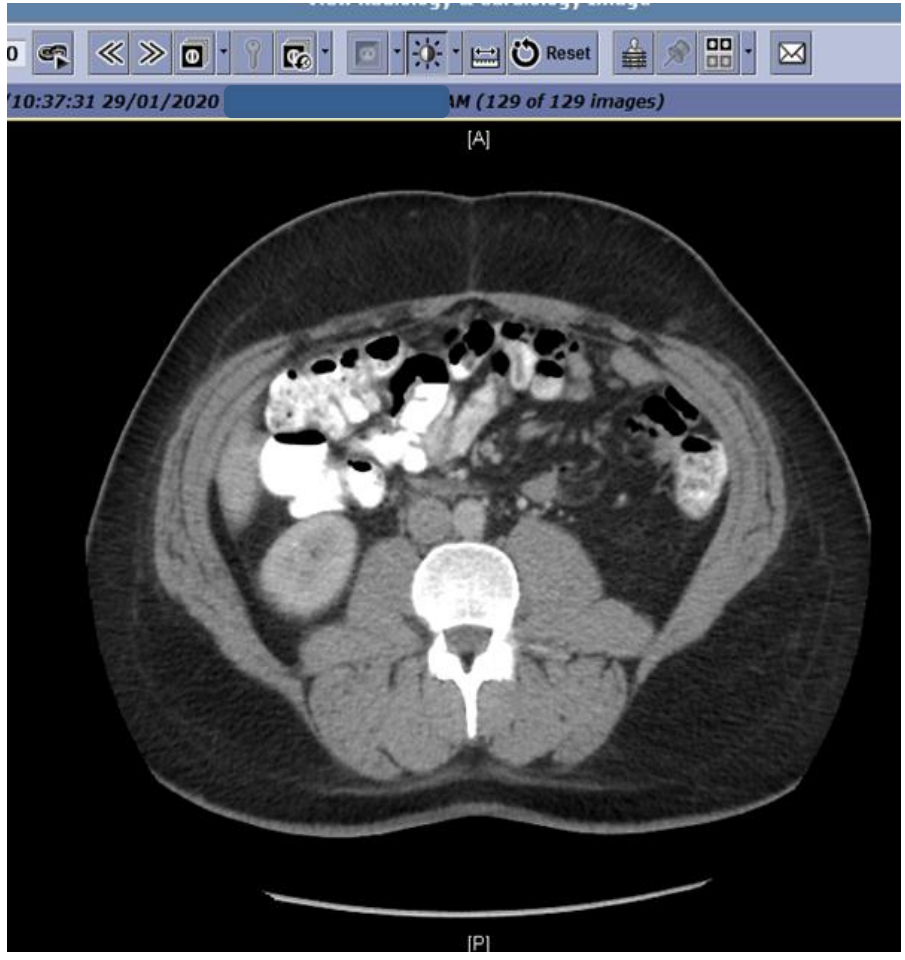

(A)

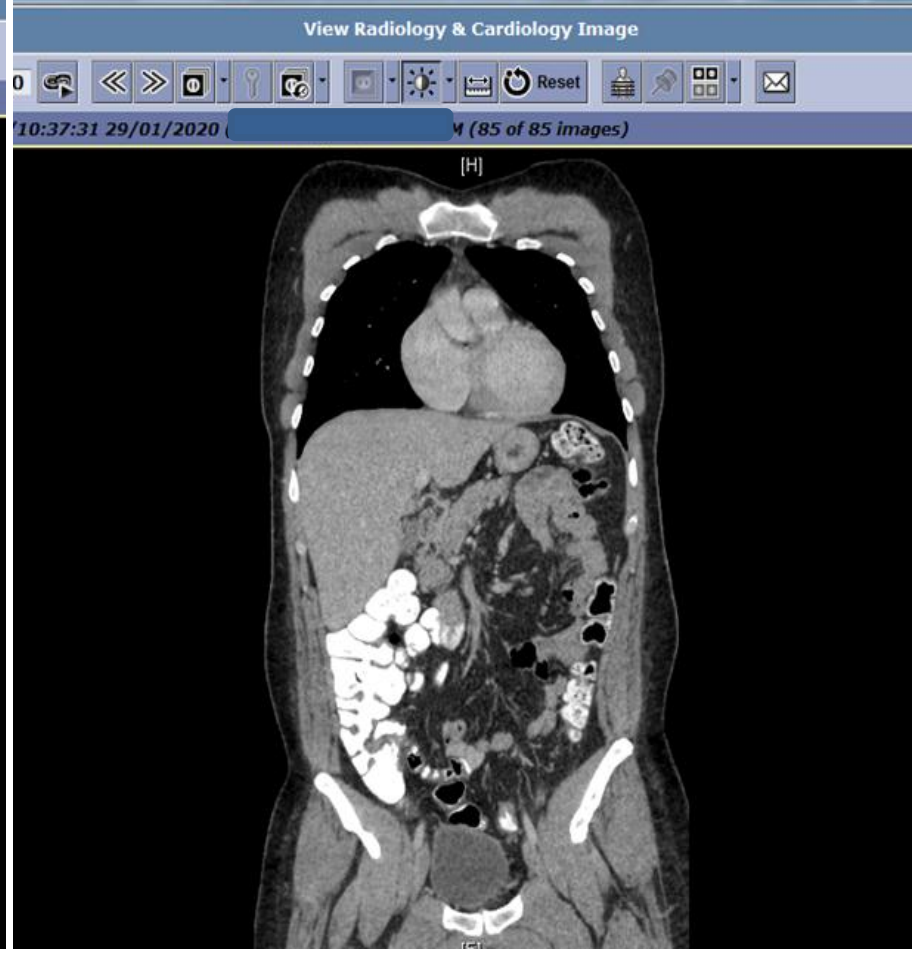

(B) 


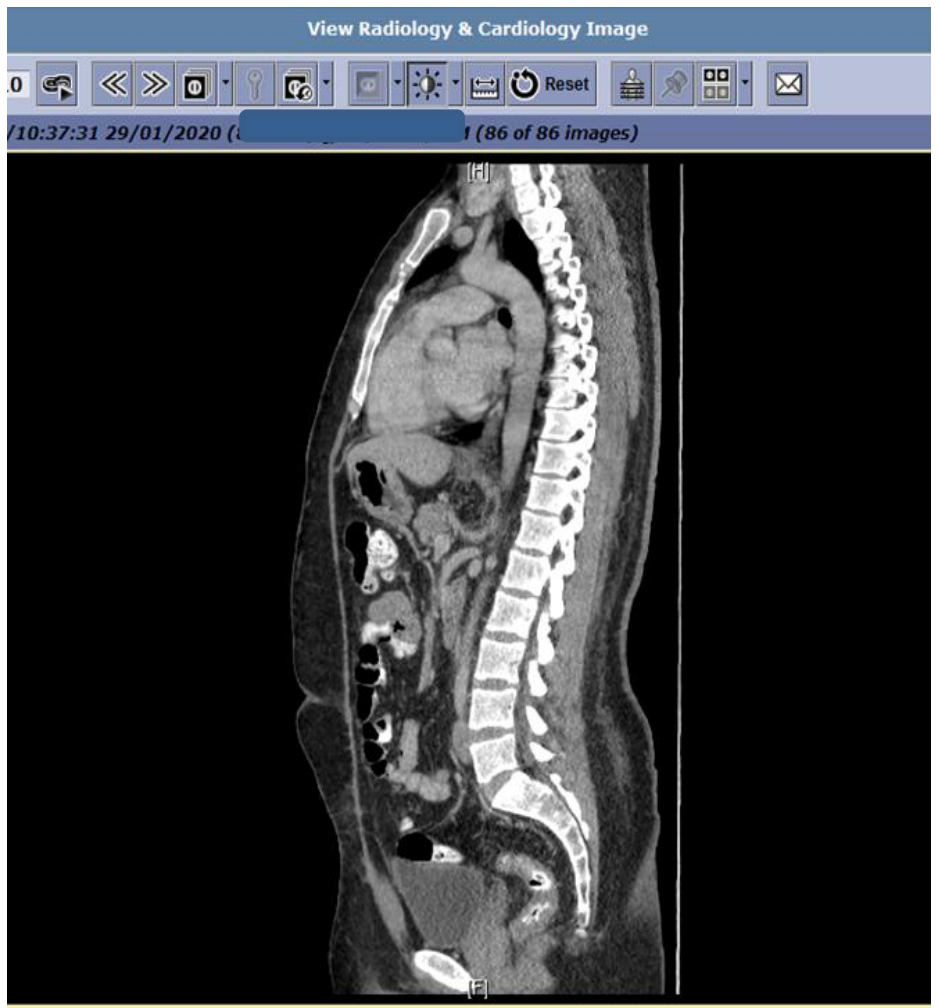

(C)

Figure3: Follow-up computed tomography scan of the abdomen 5 years later showed there is no evidence of local recurrence.

\section{Discussion}

Gastrointestinal stromal tumors are the most frequent mesenchymal tumors of the digestive system. Three distinct histologic types have been defined: spindle cell, epithelioid cell type, and mixed cell type, which comprises both spindle and epithelioid cells ${ }^{[4]}$. These tumors have been reported to range in size from $1 \mathrm{~cm}$ to $40 \mathrm{~cm}$ in diameter. The patient in our case had a huge tumor that measured approximately $42 \mathrm{~cm}$ in diameter and extended into the pelvis. A histopathology examination revealed a GIST of mixed spindle and epithelioid type, which represents approximately $10 \%$ of GISTs ${ }^{[4]}$.

The signs and symptoms of GISTs vary. In some cases, the diagnosis is fortuitous based on radiographic findings when a patient is examined for other symptoms associated with intestinal occlusion or gastrointestinal bleeding. Some patients may present with abdominal distension (as the patient did in the case being reported) and early satiety ${ }^{[5]}$. Nevertheless, about $30 \%$ of patients with GIST do not present any symptoms, and the tumor may be discovered fortuitously during an autopsy. No physical finding is considered pathognomonic for GIST. While different modalities exist for diagnosing GIST, such as barium enema, CT of the abdomen, or an abdominal ultrasound, none of these can help the clinician confirm the diagnosis. Occasionally, these diagnostic tests may be misleading in that they do not help the clinician identify the tumor.

In our patient's case, an ultrasound examination of the upper abdomen revealed a huge, cystic, septate, irregular, solid mass that displaced the liver, spleen, kidneys, bowel loops, and bladder. A spiral CT scan showed signs suggestive of a giant retroperitoneal, malignant, cystic mass, and an ultrasound-guided aspiration and analysis of the solid component of the mass revealed findings consistent with a benign cyst. Laparoscopic exploration of the abdomen revealed a huge abdominal mass with massive adhesions. Additionally, abnormally dilated vessels were identified. Due to challenges in determining the relationship between the vessels, the surrounding organs, and the tumor, a midline laparotomy was performed. The procedure revealed that the tumor extended into the pelvic cavity.

While an ultrasound of the abdomen is typically the initial radiologic investigation performed during the investigation of symptoms such as abdominal pain or distension, the detected tumor is usually large, making it challenging to determine the organ of origin. An ultrasound examination usually reveals a large, heterogeneous mass that occupies the abdomen and may show signs of necrosis, ${ }^{[6]}$ as was the case in our patient. Thus, a CT scan offers the potential to diagnose and stage the disease in most cases. Gastrointestinal stromal tumors generally have a varying density and show a heterogeneous enhancement pattern following the administration of an intravenous contrast product. Some investigators have described different levels of necrosis in the tumor, as was the case in our patient ${ }^{[7]}$. A CT scan will help the clinician obtain a quick and replicable evaluation of the primary tumor's size and its relationship with neighboring organs.

The immunologic characteristics of our patient were consistent with a GIST. Evidence shows that about $95 \%$ of GISTs are positive for CD117, which has been reported to play a major role in pathogenesis. Mutations in the gene encoding the receptor tyrosine kinase protein cause changes that induce the proliferation of cells ${ }^{[8]}$. Nevertheless, there are reports of several non-GISTs showing CD117 positivity ${ }^{[9,10]}$.

The standard of care for GISTs is surgical excision with a clear margin. There is no evidence that other treatment modalities, such as conventional chemotherapy and radiotherapy, are beneficial for GIST. According to the European Society for Medical Oncology, small tumors $<2 \mathrm{~cm}$ in diameter are low risk, and patients only need follow-up visits; however, surgical excision may be offered if the tumor increases in size or the patient becomes symptomatic ${ }^{[11]}$. The society recommends an excision biopsy for tumors $>2 \mathrm{~cm}$. Tumor reduction may be achieved by administering neoadjuvant imatinib for a minimum of six months in cases where surgery might result in functional sequelae. Given the large size of the tumor in this patient, surgical resection was deemed the most 
appropriate approach. Adjuvant therapy with imatinib was necessary to increase his cure rate and overall survival because he was at a high risk for disease recurrence. Imaging investigations are recommended for follow-ups, especially positron emission tomography scans, which are beneficial in detecting the tumor's response to targeted treatment ${ }^{[6]}$.

In conclusion, the clinical presentation of GISTs is often complex, making their diagnosis challenging, especially in patients who present with non-specific gastrointestinal symptoms. Because these tumors are potentially malignant, physicians should be familiar with their diagnosis and management. The successful management of these tumors requires a multidisciplinary approach, and regular follow-ups.

\section{Declarations}

\section{Conflict of interest}

All authors have no conflicts of interest to report.

\section{Funding Statement}

All authors did not receive funding for this paper.

\section{Consent}

Informed consent was obtained from the patient prior to the publication of this report.

\section{List of abbreviation:}

GIST: Gastrointestinal stromal tumors

CT: computed tomography

CEA: carcinoembryonic antigen

AFP: alpha-fetoprotein

\section{References}

[1] Fülöp E, Marcu S, Milutin D, Borda A. Gastrointestinal stromal tumors: review on morphology, diagnosis and management. Romanian J Morphol Embryol Rev Roum Morphol Embryol. 2009;50(3):319-26.
[2] Van der Zwan SM, DeMatteo RP. Gastrointestinal stromal tumor: 5 years later. Cancer. 2005 Nov 1;104(9):1781-8.

[3] Zhou L, Liu C, Bai J-G, Wei J-C, Qu K, Tian F, et al. A rare giant gastrointestinal stromal tumor of the stomach traversing the upper abdomen: a case report and literature review. World J Surg Oncol. 2012 Apr 27;10(1):66.

[4] Miettinen M, Lasota J. Gastrointestinal stromal tumors: review on morphology, molecular pathology, prognosis, and differential diagnosis. Arch Pathol Lab Med. 2006 Oct;130(10):1466-78.

[5] Sepe PS, Brugge WR. A guide for the diagnosis and management of gastrointestinal stromal cell tumors. Nat Rev Gastroenterol Hepatol. 2009 Jun;6(6):363-71.

[6] Gong J, Kang W, Zhu J, Xu J. CT and MR imaging of gastrointestinal stromal tumor of stomach: a pictorial review. Quant Imaging Med Surg. 2012 Dec;2(4):274-9.

[7] King D. The radiology of gastrointestinal stromal tumours (GIST) [Internet]. Vol. 5, Cancer imaging: the official publication of the International Cancer Imaging Society. Cancer Imaging; 2005 [cited 2020 Nov 23]. Available from: https://pubmed.ncbi.nlm.nih.gov/16361144/

[8] Hirota S, Isozaki K, Moriyama Y, Hashimoto K, Nishida $\mathrm{T}$, Ishiguro $\mathrm{S}$, et al. Gain-of-function mutations of c-kit in human gastrointestinal stromal tumors. Science. 1998 Jan 23;279(5350):577-80.

[9] Medeiros F, Corless CL, Duensing A, Hornick JL, Oliveira AM, Heinrich MC, et al. KIT-negative gastrointestinal stromal tumors: proof of concept and therapeutic implications. Am J Surg Pathol. 2004 Jul;28(7):889-94.

[10] Medinger M, Kleinschmidt M, Mross K, Wehmeyer B, Unger C, Schaefer H-E, et al. c-kit (CD117) expression in human tumors and its prognostic value: an immunohistochemical analysis. Pathol Oncol Res POR. 2010 Sep;16(3):295-301.

[11] Casali PG, Abecassis N, Bauer S, Biagini R, Bielack S, Bonvalot S, et al. Gastrointestinal stromal tumours: ESMO-EURACAN Clinical Practice Guidelines for diagnosis, treatment and follow-up. Ann Oncol. 2018 Oct;29:iv68-78. 